\title{
Actitudes y prácticas sobre lactancia materna en puérperas de un hospital público de Lima, Perú
}

\author{
Christian R. Mejia ${ }^{1,2}$, Matlin M. Cárdenas $3, a$, Onice J. Cáceres ${ }^{3, a}$, Katerine M. García- \\ Moreno $^{4, a}$, Araseli Verastegui-Díaz $3,5, a$, Dante M. Quiñones-Laveriano. 3,5,a
}

${ }^{1}$ Escuela de Medicina, Universidad Peruana de Ciencias Aplicadas -UPC. ${ }^{2}$ Asociación Médica de Investigación y Servicios en Salud. ${ }^{3}$ Facultad de Medicina Humana, Universidad Ricardo Palma. ${ }^{4}$ Sociedad Científica de Estudiantes de Medicina de la Universidad Ricardo Palma. ${ }^{5}$ Instituto de Investigación en Ciencias Biomédicas, Universidad Ricardo Palma. Lima, Perú.

a Alumno/a de Medicina, Facultad de Medicina Humana, Universidad Ricardo Palma. Lima, Perú.

\section{RESUMEN}

Antecedentes: La lactancia materna es el alimento perfecto, pero las madres deben tener adecuadas prácticas para hacerlo de manera correcta. Objetivo: Determinar las actitudes y prácticas sobre lactancia en puérperas y sus factores socio-perinatales asociados. Métodos: Estudio transversal analítico desarrollado entre octubre y diciembre del 2012. Se utilizó un cuestionario previamente validado de respuesta nominal, auto-aplicada con preguntas de opción múltiple sobre actitudes y prácticas de la lactancia materna. Se obtuvo una muestra no probabilística de las mujeres en la unidad obstétrica del Hospital Nacional María Auxiliadora. Se usó la regresión lineal para el análisis bi y multivariado, obteniendo los coeficientes crudos y ajustados con sus intervalos de confianza al 95\%. Resultados: Participaron 256 puérperas, la mediana de edades fue de 24,5 años (rango 18-46 años), el 25\% tuvieron 7 buenas respuestas de las 20 planteadas. Se encontró una correlación positiva entre mayor número de respuestas correctas y las variables de edad $(p=0,002$, Coeficiente: 0,1$)$, número de controles prenatales $(p=0,031$, Coeficiente: 0,1$)$ y una correlación negativa con la información recibida por otra persona que no es profesional de la salud $(p=0,033$, Coeficiente:-1,2). Conclusiones: Existe una asociación entre las actitudes y prácticas sobre lactancia materna según algunos factores socio-perinatales, siendo esto importante para la planificación de programas de capacitación. Estos resultados coinciden con otras investigaciones que demuestran que hay un incremento de conocimiento a mayor experiencia de la gestante.

\section{PALABRAS CLAVE: Lactancia materna, actitudes y prácticas, período post parto, Perú}

\section{SUMMARY}

Background: Breastfeeding is the perfect food, but mothers should have appropriate practices to do it right. Aims: Determine the attitudes and practices on breastfeeding in postpartum women and their socio-perinatal factors associated. Methods: Cross-sectional study conducted between October and December 2012. We used a previously validated questionnaire self applied nominal response with multiple choice questions on attitudes and practices of breastfeeding. We obtained a non-probability sample of women in the birthing of Hospital Nacional María Auxiliadora. Linear regression was used for bi and multivariate analysis, obtaining the crude and adjusted coefficients with confidence intervals at $95 \%$. Results: Participants 256 postpartum women, median age was 24.5 years (range $18-46$ years), $25 \%$ had 7 good answers of the 20 raised. We found a positive correlation between higher number of correct responses and the variables of 
age ( $p=0.002$, coefficient: 0.1$)$, number of prenatal visits $(p=0.031$, coefficient: 0.1$)$ and a negative correlation with the information received by another person who is not healthcare $(p=0.033$, coefficient: -1.2$)$. Conclusion: There is an association between attitudes and practices on breastfeeding by socio-perinatal factors, this being important for planning training programs. These results are consistent with other research showing that there is a greater increase in knowledge of pregnant women experience.

\section{KEYWORDS: Breast feeding, attitudes and practices, postpartum period, Peru}

\section{INTRODUCCIÓN}

La lactancia materna es definida según la Organización Mundial de la Salud (OMS) como la forma ideal de aportar a los niños pequeños los nutrientes que necesitan para un crecimiento y desarrollo saludable, y la recomienda como el alimento perfecto para el recién nacido $(1,2)$. Se sabe que este periodo es fundamental para el niño, pues de ello depende el comienzo de su desarrollo y la menor tendencia a presentar enfermedades infecciosas (3-5). Es por ello que existe la "Estrategia Mundial para la Alimentación del Lactante y del Niño Pequeño", la cual se orienta en brindar la promoción y el apoyo a la lactancia materna y la orientación sobre la ejecución de la misma (6).

Estudios realizados en América Latina muestran que existen prácticas sub-óptimas de lactancia materna (7-9), principalmente en cuanto al uso de leches artificiales, líquidos al recién nacido, duración reducida de la lactancia e inadecuada elección del momento de introducción de los alimentos complementarios (10-12). Así mismo, reportes en realidades similares a la nuestra muestran que una gran población se ve afectada debido a una inadecuada práctica de la lactancia por parte de la puérpera, trayendo consecuencias adversas para ella y el neonato (13-14). Pudiendo ser esto causado por algunas creencias que tienen las madres acerca de la lactancia materna, que si bien varían según las costumbres y tradiciones de cada cultura, muchas de estas son erróneas y generan consecuencias que atentan esencialmente contra la buena alimentación del recién nacido $(1,10,12)$.

En nuestro país los estudios que abordan temas de lactancia materna son escasos y antiguos. Por lo anteriormente mencionado, el objetivo del estudio fue determinar las actitudes y prácticas sobre lactancia materna en puérperas.

\section{PACIENTES Y MÉTODOS}

Diseño y población de estudio: Estudio de corte transversal analítico. Considerando las actitudes y prácticas con respecto a la lactancia materna en un grupo de puérperas del Hospital Nacional María Auxiliadora, institución pública de Lima, Perú.
Se incluyó a mujeres mayores de edad en etapa de puerperio. Previo a su participación se le explicó el objetivo del estudio y que su participación era voluntaria, las que aceptaron participar en el estudio y otorgaron su consentimiento verbal se tomaron en cuenta para el encuestado. Se excluyó a mujeres en etapa de gestación y que presentaban alguna alteración del sensorio (menos de $2 \%$ de exclusión).

Se utilizó un muestreo por conveniencia, para obtener el tamaño mínimo muestral se usó $50 \%$ como valor máximo de respuestas incorrectas, con un nivel de confianza del 95\% una precisión de 7\% y sumando un $10 \%$ para los rechazos, se obtuvo un tamaño mínimo muestral de 216 puérperas.

Instrumentos y variables: El estudio se realizó mediante el empleo de una encuesta auto-aplicada, la cual fue elaborada con el objetivo de medir las actitudes y prácticas sobre lactancia materna en puérperas. El instrumento usó una escala de respuesta nominal tipo test de potencia, el cual fue construido teniendo en cuenta cuales eran las prácticas más populares sobre la lactancia materna, dicha elaboración estuvo a cargo de un grupo de estudiantes de una universidad particular (como parte de un curso académico). Los temas abordados fueron actitudes y prácticas sobre: cambio de peso corporal materno (dar de lactar aumentaba de peso a la madre); alimentación (alimentos que influyen en la producción de leche, o que estén prohibidos durante la etapa de lactancia como el consumo de ají, infusiones o alimentos fríos); y en la salud del recién nacido (influencia de la leche materna en caso de episodios patológicos como la diarrea), o si la leche materna puede llegar a causar daño en el lactante, también se evaluó el tema de sexualidad (si la lactancia tenía alguna relación con la vida sexual de la madre).

Para la elaboración de las preguntas se contó con la colaboración de dos pediatras con más de 10 años de experiencia, quienes sugirieron 37 preguntas sobre actitudes y prácticas en lactancia materna y 11 preguntas que medirían variables intervinientes. Estas preguntas fueron evaluadas en cuanto a su pertinencia (fondo) y la comprensión (forma) por 4 médicos de un hospital público, cuya evaluación se realizó con una escala del $0-10$, 
sólo se consideraron en el cuestionario final las preguntas con un promedio mayor o igual a ocho. Posterior a esto se realizó el cuestionario a 5 estudiantes de medicina, quienes evaluaron la comprensión de la preguntas, se usó la misma escala que la usada por los médicos. Se eliminó 9 preguntas que obtuvieron las más bajas notas. Por último, se realizó un proyecto piloto, donde se evaluó las respuestas de 54 puérperas de la misma institución donde se aplicaría el cuestionario. Seis preguntas obtuvieron $20 \%$ o más de respuestas con la alternativa "no sé", por lo que fueron eliminadas, resultando una encuesta final de 20 preguntas.

Procedimientos y ética: Se solicitó permiso para realizar el trabajo en el Hospital Nacional María Auxiliadora. Una vez obtenido el permiso se acudió a las salas de puerperio a las madres que aceptaran participar en el estudio y que cumplían los criterio de inclusión. Las encuestas fueron pasadas a una base de datos por el equipo investigador, donde fueron depuradas antes del análisis. El protocolo fue aprobado por el comité de ética del Hospital Nacional Docente Madre Niño San Bartolomé.

Análisis de datos: La base se creó usando el programa Microsoft Excel para Windows 2010. Los datos fueron procesados en el paquete estadístico STATA 11,1 (StataCorp LP, College Station, TX, USA). En el análisis descriptivo se evaluó las variables numéricas con medias y desviación estándar; se analizó las variables categóricas utilizando frecuencias y porcentajes. Para la estadística inferencial se usó la nota que obtuvieron las puérperas según las respuestas correctas del test de 20 preguntas, usando la nota como variable cuantitativa se analizó los factores relacionados en el análisis bi y multivariado con la regresión lineal, con intervalos de confianza al 95\% (IC95\%). Se consideró un valor $\mathrm{p}<0,05$ como significativamente estadístico.

\section{RESULTADOS}

Participaron 256 gestantes que cumplían con los criterios de selección del estudio, 240 (93,8\%) vivían en Lima, la mediana de edades fue 24,5 años (rango: 18-46 años), así mismo, 15 (5,9\%) contaban con educación primaria o eran analfabetas, $207(80,9 \%)$ se encontraban en estado de pobreza, $103(40,2 \%)$ que ya habían dado de lactar como antecedente. Del total de encuestadas, 160 $(62,5 \%)$ recibieron información sobre lactancia materna, de las cuales $22(8,6 \%)$ recibieron la información por un médico y $83(32,4 \%)$ recibieron esta información en un hospital.

La nota promedio que obtuvieron de las preguntas sobre lactancia materna fue 8,9 (desviación estándar: $\pm 2,23$ ), la nota máxima fue obtenida por una gestante, con 15 puntos y $25 \%$ de las puérperas obtuvieron una nota $\leq 7$ puntos; el $50 \%$ de las puérperas obtuvieron una nota $\leq 9$ puntos y
$75 \%$ de las puérperas una nota $\leq 10$ puntos. La importancia de la forma del pezón fue la pregunta con mejores porcentajes de respuesta, habiendo respondido correctamente 242 (96,0\%) puérperas, así mismo, $12(5,1 \%)$ puérperas respondieron que dar de lactar engorda a la madre. Las frecuencias de respuesta por cada pregunta se presentan en la Tabla I.

Las variables que resultaron significativas en el análisis bivariado (Tabla II), pasaron al modelo multivariado; se mantuvo asociado el número de respuestas correctas según la edad de la gestante $(p=0,002$, Coef $=0,06$, IC95\% 0,02-0,09), cantidad de controles prenatales $(p=0,031$, Coef $=0,10$, IC95\% 0,01-0,20) en las cuales hubo una correlación positiva mientras que en la información recibida por otra persona que no es personal de salud $(p=0,33$, Coef $=-1,20$, IC95\% -2,31- $(-0,10))$ se encontró una correlación negativa (Tabla III).

\section{DISCUSIÓN}

La lactancia materna y la adecuada nutrición al recién nacido constituyen pilares fundamentales de la promoción de la salud, por su importancia en la prevención de diversas enfermedades (16-19), esta es considerada un acto natural y fisiológico que se ha practicado desde inicios de los tiempos, a lo largo del cual han aparecido diversas actitudes y prácticas que afectan la adecuada alimentación del neonato (19-22). Encontramos que una de cada 4 mujeres encuestadas obtuvo una nota $\leq 7$, lo cual es un signo de alarma ante la falta de conocimiento de las madres. Es de resaltar que las encuestadas fueron atendidas en una sede hospitalaria, siendo ellas las que deberían contar con un mejor conocimiento sobre este tema, con respecto a las que viven en lugares alejados de las grandes ciudades. Esto debería ser tomado en cuenta por las instituciones correspondientes, para realizar intervenciones educativas que mejoren los niveles de conocimiento, lo que ha sido implementado en distintas realidades, donde demuestran que intervenciones educativas constantes mejoran en hasta un $100 \%$ los conocimientos en las gestantes $(7,19,23)$.

Se encontró que el aumento de edad es un factor asociado al mayor número de respuestas correctas, estos resultados coinciden con investigaciones que demuestran que hay un incremento de conocimiento al aumentar la edad de la gestante $(10,24)$. Como el realizado en Cuba, en donde las mujeres con más de 35 años resultaron tener mejores conocimientos sobre lactancia materna, aproximadamente 6 de cada 10 madres adolescentes tuvieron conocimientos insuficientes sobre lactancia materna; así mismo, una cuarta parte tenía conocimientos suficientes y menos de la quinta parte amplios conocimientos (19). 


\section{FRECUENCIA DE RESPUESTAS CORRECTAS ACERCA DE LAS ACTITUDES Y PRÁCTICAS DE LACTANCIA MATERNA EN PUÉRPERAS DE UN HOSPITAL PÚBLICO EN LIMA}

\begin{tabular}{|c|c|c|}
\hline Pregunta de cuestionario & $\mathrm{n}$ & $\%$ \\
\hline ¿Hay que darle de lactar al niño cada vez que lo pida? & 199 & 78,7 \\
\hline ¿La forma del pezón es importante para la lactancia? & 242 & 96,0 \\
\hline ¿Los niños alimentados con leche materna suelen ser más inteligentes? & 176 & 76,2 \\
\hline Si su abuela, madre o hermanas no tuvieron mucha leche ¿usted tampoco la tendrá? & 26 & 11,8 \\
\hline ¿Dar de lactar engorda a la madre? & 12 & 5,1 \\
\hline ¿Los senos se deforman por dar de mamar? & 115 & 49,2 \\
\hline Si Ud. consume alimentos fríos, ¿debería dar de lactar? & 55 & 22,6 \\
\hline ¿La cantidad de leche materna que se produce depende del tamaño del seno? & 53 & 23,5 \\
\hline $\begin{array}{l}\text { La leche que la madre produce en los primeros días después del parto, ¿debería ser des- } \\
\text { echada por que es dañina para su bebe? }\end{array}$ & 220 & 94,8 \\
\hline Si Ud. está dando de lactar, ¿debería comer más de lo acostumbrado? & 124 & 49,6 \\
\hline ¿Hay alimentos que aumentan la producción de leche? & 26 & 11,0 \\
\hline ¿Los disgustos de la madre se transmiten al niño por la lactancia materna? & 51 & 21,6 \\
\hline ¿La madre que da de lactar puede comer aji? & 49 & 22,3 \\
\hline Si el niño se llena de gases después de lactar, ¿fue por algún alimento que Ud. consumió? & 95 & 41,7 \\
\hline ¿Si usted ingiere poco líquido disminuirá la producción de leche? & 64 & 26,7 \\
\hline ¿Si su niño lacta muy seguido se enfermará del estómago? & 225 & 94,9 \\
\hline ¿Dar de mamar hace que la madre pierda calcio? & 136 & 62,4 \\
\hline ¿Lo ideal es que el bebé tome primero toda la leche de un seno y luego del otro? & 100 & 42,6 \\
\hline ¿Después de 6 meses la leche materna ya no alimenta? & 136 & 59,7 \\
\hline ¿La actividad física y el trabajo disminuyen la producción de leche? & 176 & 85,4 \\
\hline
\end{tabular}




\section{Tabla II}

ANÁLISIS BIVARIADO DEL NÚMERO DE RESPUESTAS CORRECTAS SEGÚN CARACTERÍSTICAS MATERNAS EN PUÉRPERAS DE UN HOSPITAL PÚBLICO EN LIMA

\begin{tabular}{lccc}
\hline & Coef. & Valor $\mathrm{p}$ & IC 95\% \\
\hline Edad & 0,1 & 0,005 & $0,02-0,95$ \\
Vive en Lima & $-0,3$ & 0,604 & $-1,44-0,84$ \\
Hijos & 0,2 & 0,131 & $-0.06-0,44$ \\
Educación básica & $-0,3$ & 0,585 & $-1,50-0,85$ \\
Cantidad controles prenatales & 0,1 & 0,034 & $0,01-0,20$ \\
Lactantes previos & 0,1 & 0,737 & $-0,20-0,29$ \\
Recibió información & 0,3 & 0,249 & $-0,23-0,90$ \\
Periodo en el que recibió la información: & & & \\
Antes de la gestación & $-0,1$ & 0,866 & $-0,90-0,76$ \\
Durante la gestación & 0,3 & 0,221 & $-0,21-0,90$ \\
Después de la gestación & $-0,5$ & 0,260 & $-1,48-0,40$ \\
Persona de la que recibió la información: & & & \\
Médico & 0,9 & 0,071 & $-0,7-1,88$ \\
Obstetra & 0,5 & 0,085 & $-0,07-1,11$ \\
Enfermera & $-0,1$ & 0,755 & $-0,81-0,59$ \\
Nutricionista & $-0,2$ & 0,742 & $-1,42-1,01$ \\
Otros & $-1,2$ & 0,043 & $-2,30-(-0,37)$ \\
Lugar donde recibió la información: & & & \\
Hospital & 0,6 & 0,050 & $-0,01-1,16$ \\
Posta & 0,1 & 0,954 & $-0,59-0,63$ \\
Otros: & $-0,4$ & 0,520 & $-1,73-0,88$ \\
Estado de pobreza & 0,3 & 0,419 & $-0,41-0,99$ \\
\hline
\end{tabular}

Coef.: Coeficiente. IC 95\%: Intervalos de confianza al 95\%, con regresión lineal bivariado.

Tabla III

ANÁLISIS MULTIVARIADO DEL NÚMERO DE RESPUESTAS CORRECTAS SEGÚN CARACTERÍSTICAS MATERNAS EN PUÉRPERAS DE UN HOSPITAL PÚBLICO EN LIMA

\begin{tabular}{lccc}
\hline & Coef. & Valor $\mathrm{p}$ & IC 95\% \\
\hline Edad & 0,1 & 0,002 & $0,02-0,09$ \\
Cantidad de controles prenatales & 0,1 & 0,031 & $0,01-0,20$ \\
Información recibida por otra persona que no es personal de salud & $-1,2$ & 0,033 & $-2,31-(-0,10)$
\end{tabular}

Coef.: Coeficiente. IC 95\%: Intervalo de confianza al 95\%, con regresión lineal multivariada. 
El incremento en el número de respuestas correctas estuvo asociado con un mayor número de controles prenatales, al tener un mayor número de chequeos médicos la gestante tiene mayor conocimiento sobre las correctas prácticas que se debe realizar en el amamantamiento; estudios demuestran que a mayor cantidad de controles aumenta el conocimiento en lactancia materna, pero difiere de un estudio realizado en Kenia, que encontró un $59 \%$ de madres que habían tenido controles prenatales, posiblemente porque dicho estudio tomó a la variable de controles prenatales presente o ausencia, de modo que no se puede determinar la influencia del número de controles prenatales (25).

Se encontró una correlación negativa entre la asociación de numero de respuestas correctas e información recibida por otra persona que no sea personal de salud. Esta situación puede tener un efecto negativo sobre la práctica de la lactancia materna, en la cual se observa que las gestantes toman con mayor importancia la información proporcionada por familiares, amigos, etc., dejando de lado aquella información brindada por un profesional de la salud, cualquier información que sea incorrecta, incompleta o falta de evidencia científica puede llevar a una inadecuada alimentación del neonato (26-28).

No se encontró una asociación entre el mayor número de respuestas correctas y educación básica. Sin embargo muchos autores han demostrado que existe un predominio de madres con niveles de estudios superiores, y que a medida que aumenta el nivel de escolaridad tienen un mejor conocimiento sobre lactancia $(7,25,29,30)$.

Una de las limitaciones del estudio fue el sesgo de selección, ya que no se realizó un muestreo aleatorio a las madres encuestadas, sin embargo, nos basamos en un tamaño muestral mayor al requerido y con una buena precisión, por lo que creemos que los resultados se aproximan a la realidad, además, al ser uno de los primeros reportes en el tema es importante porque brinda datos primarios de las actitudes y prácticas de la lactancia materna.

\section{CONCLUSIÓN}

Existe una asociación entre las actitudes y prácticas sobre lactancia materna según edad de la madre, el número de controles prenatales que tuvieron durante su gestación y la información recibida por otra persona que no es personal de la salud. Teniendo las dos primeras una correlación positiva y la última una correlación negativa.

El estudio de los temas relacionados con la lactancia materna necesita muchas más indagaciones, que tengan un adecuado tamaño muestral y que además abarquen otros factores que influyen. A los profesionales de la salud se les recomienda dar una mejor explicación sobre el tema en los controles prenatales encaminada a incrementar y afianzar los conocimientos sobre el tema. Esperamos que en base a estos resultados las autoridades correspondientes generen políticas de intervención que ayuden a mejorar la realidad encontrada.

\section{REFERENCIAS}

1. Puerta SA, López RA. Lactancia materna basada en la evidencia. Garnata 2012;91(22):41-5. Disponible en: http://www.coegr.es/images/documentos/publicaciones/garnata/garnata-22.pdf

2. Borda GC, Lipa LB, Gonzáles J, Villacampa SAC. Beneficios de la leche y lactancia materna como factor importante del crecimiento y desarrollo del niño y su relación con el órgano de la boca. Odontol Sanmarquina. 2014;8(1):46-50. Disponible en: http://revistasinvestigacion.unmsm.edu.pe/index.php/odont/ article/viewFile/3383/4556.

3. Rodríguez-García J, Acosta-Ramírez N. Factores asociados a la lactancia materna exclusiva en población pobre de áreas urbanas de Colombia. Rev Salud Publica 2008;10(1):71-84. Disponible en: http://www. scielosp.org/pdf/rsap/v10n1/v10n1a07

4. Aguirre AGH, Tapia JR, Aceves RS, Bautista VMH. El sistema inmune neonatal y su relación con la infección. Pediátricas 2013;22(3):101-13. Disponible en: http://www.medigraphic.com/pdfs/alergia/al-2013/ al133c.pdf

5. Vicente MPS. Los grupos de apoyo a la lactancia materna como iniciativas de promoción de la salud en la comunidad: un estudio descriptivo en la Región de Murcia [Internet]. 2014. Disponible en: http://digitum. um.es/xmlui/handle/10201/37724

6. OPS. Semana Mundial de la Lactancia Materna, 2012. Comprendiendo el Pasado - Planificando el Futuro. Celebración del $10^{\circ}$ aniversario de la Estrategia Mundial para la Alimentación del Lactante y del Niño Pequeño de la OMS/UNICEF. Disponible en: www. paho.org/alimentacioninfantil

7. Rodríguez JE, Ramírez JA, Domínguez BR, Basulto AG. Intervención educativa sobre lactancia materna. Revista Archivo Médico de Camagüey. abril de 2010;14(2). Disponible en: http:// scielo.sld.cu/scielo.php?script=sci_arttext\&pid =S1025-02552010000200009

8. OMS, UNICEF. Asamblea Mundial de la Salud. Nutrición del lactante y del niño pequeño: estrategia mundial para la alimentación del lactante y del niño pequeño. Informe de la Secretaría. Ginebra: OMS; 2002. Disponible en: https://www.ministeriodesalud. go.cr/gestores_en_salud/lactancia/CNLM_estrategia_mundial.pdf

9. Forero $\mathrm{Y}$, Rodríguez SM, Isaács MA, Hernández JA. La lactancia materna desde la perspectiva de madres adolescentes de Bogotá. Biomédica 2013;33(4):55463. Disponible en: http://www.scielo.org.co/pdf/bio/ v33n4/v33n4a09.pdf

10. Sosa MMF, Condori HFF. Nivel de conocimiento en lactancia materna y su relación con factores socioculturales en puérperas del IEMP de octubre a diciembre del 2005. [Lima-Perú]: Universidad Mayor de San Marcos; 2006. Disponible en: http://cybertesis. unmsm.edu.pe/bitstream/cybertesis/2975/1/Ferro_ sm.pdf 
11. Oyaque CEC. Prácticas y conocimientos, creencias y tabús alimentarios que influyen en el estado nutricional de las mujeres gestantes y lactantes atendidas en el centro de salud de Huachi Chico durante el período junio-noviembre del 2013. 2014. [Internet]. 2014. Disponible en: http://repositorio.uta.edu.ec/handle/123456789/7660

12. Macías SM, Rodríguez S, Ronayde de Ferrer PA. Patrón de alimentación e incorporación de alimentos complementarios en lactantes de una población de bajos recursos. Rev Chil Nutr 2013;40(3):235-42.

13. Bautista LE. Factores asociados al inicio de la lactancia materna en mujeres dominicanas. Rev Panam Salud Publica/Pan Am J Public Health. 1997;1(3):200-7.

14. Flores LAC. Factores de riesgo perinatales, sociales y alimentarios para el desarrollo de sobrepeso y obesidad en lactantes de un año de edad. [Internet]. 2014. Disponible en: http://ri.uaq.mx/bitstream/123456789/1008/1/RI000532.pdf

15. Dalmao AR, Sotomayor IG, Céspedes YYG, González MV. Factores de riesgo asociados a las infecciones respiratorias agudas en lactantes, Veguitas 2013. Multimed Rev Médica Granma. 2014;18(1). Disponible en: http://www.medigraphic.com/pdfs/multimed/ mul-2014/mul141f.pdf.

16. Alzate-Meza MC, Arango C, Castaño-Castrillón JJ, Henao-Hurtado AM, Lozano-Acosta MM, MuñozSalazar G, et al. Lactancia materna como factor protector para enfermedades prevalentes en niños hasta de 5 años de edad en algunas instituciones educativas de Colombia, 2009. Rev Colomb Obstet Ginecol 2011;62(1):57-63.

17. Ruiz JMP, Ibabe MCI, Herreros JMP, León MTM, Campoy F. Lactancia materna y desarrollo cognitivo; valoración de la respuesta a la interferencia mediante el «test de los 5 dígitos». Nutr Hosp. 2014;29(4):8527. Disponible en: http://www.aulamedica.es/gdcr/index.php/nh/article/view/7211

18. Jaramillo JB, Arias LPB, Castrillón JJC, Rico OM, Obando VM, Villada HAO. La lactancia materna y su relación con patologías prevalentes en la infancia en jardines infantiles de la ciudad de Manizales (Colombia), 2013. Archivos de Medicina 2014;14(1):29-43. Disponible en: http://www.redalyc. org/pdf/2738/273832164004.pdf.

19. Carrasco-Loyola ML, Villena-Sarmiento RS, PachasBarrionuevo FM, Sánchez-Huamán YD. Lactancia materna y hábitos de succión nutritivos y no nutritivos en niños de 0-71 meses de comunidades urbano marginales del cono norte de Lima. Rev Estomatol Herediana 2009;19(2):83-90. Disponible en: http://www. upch.edu.pe/vrinve/dugic/revistas/index.php/REH/ article/view/1826

20. Colina JAD, Colina MD, Martínez LF, Sarriá J. Educación prenatal y lactancia materna. Mediciego 2006;12(1). Disponible en: http://bvs.sld.cu/revistas/ mciego/vol12_01_06/articulos/a9_v12_0106.html.
21. Pérez RRG, Linares DB. Deseos, aptitudes y conocimientos de las gestantes sobre lactancia materna: algunos factores asociados. Revista de Ciencias Médicas La Habana. 2011;17(2):204-15. Disponible en: http://revcmhabana.sld.cu/index.php/rcmh/article/ view/522/html_3.

22. Stuebe MA. Formas de facilitar que la mujer alcance sus metas de lactancia materna. Obstet Gynecol. 2014;123:643-52. Disponible en: http://journals.Iww. com/greenjournal/Documents/Mar2014_StuebeCES_ Translation.pdf

23. Remigio $R$, Gorrita $P$, Rodríguez $Y R$, Hernández ER, Martínez MO. Morbilidad y lactancia materna exclusiva en el primer semestre en relación con una intervención educativa. Revista De Ciencias Médicas. La Habana. 2015; 21(3):526-539. Disponible en: http://new.medigraphic.com/cgi-bin/resumen. cgi?IDARTICULO $=63773$

24. Sánchez MRG, Cordero MEA, Concepción MF. Nivel de conocimientos sobre lactancia materna en un grupo de embarazadas. Revista de Ciencias Médicas La Habana. 2010;16(2):136-45. Disponible en: http://revcmhabana.sld.cu/index.php/rcmh/article/ view/489/827

25. Perumal N, Cole DC, Ouédraogo HZ, Sindi K, Loechl $\mathrm{C}$, Low $\mathrm{J}$, et al. Health and nutrition knowledge, attitudes and practices of pregnant women attending and not-attending ANC clinics in Western Kenya: a cross-sectional analysis. BMC Pregnancy Childbirth 2013;13(1):146.

26. Fonseca-Machado $M$ de O, Haas VJ, Stefanello J, Nakano AMS, Gomes-Sponholz F. [Breastfeeding: knowledge and practice]. Rev Esc Enferm USP. agosto de 2012;46(4):809-15. Disponible en: http://www. redalyc.org/pdf/3610/361033319004_2.pdf

27. Hernández L, Vásquez ML. Practices and beliefs about exclusive breastfeeding by women living in Commune 5 in Cali, Colombia. Colombia Médica 2010;41(2):161-70. Disponible en: http://colombiamedica.univalle.edu.co/index.php/comedica/article/ view/698/798

28. Rodríguez VX, Morawicki PM. Reflexiones en torno a una propuesta didáctica sobre Alimentación y Nutrición Materno-Infantil en un contexto de Educación No Formal. Revista de Educación en Biología.2014;17(1):135-44. Disponible en: www.revistaadbia.com.ar/ojs/index.php/adbia/article/download/92/ pdf

29. Suárez LR, Cosme BP. Intervención de enfermería para la capacitación sobre lactancia materna. Rev Cubana Enfermer. 2004;20(1):1-1. Disponible en: http://scielo.sld.cu/scielo.php?script=sci_arttext\&pid $=$ S0864-03192004000100002

30. Niño MR, Silva EG, Atalah SE. Factores asociados a la lactancia materna exclusiva. Rev Chil Pediatr. 2012; 83 (2):161-9. 